\title{
Mathematical simulation of transient operation modes of an electric drive of a centrifugal pump for a slurry pipeline
}

\author{
Alexander Korzhev, Olga Bolshunova, Irina Voytyuk* and Anna Vatlina \\ Saint-Petersburg Mining University, St. Petersburg, Russian Federation
}

\begin{abstract}
To develop new control algorithms for controlling slurry pumps, it is necessary to have a reliable mathematical model of their operation, convenient for computer simulation implementation. Therefore, the paper discloses a mathematical model of the joint operation of a centrifugal pump with a controlled asynchronous motor drive and a slurry pipeline. The results of transient modes computer simulation, carried out via the xcos application of the scilab software, are presented. This software is a freeware one and, therefore, available free of charge to most researchers. The paper provides an example of verification of the adequacy and performance of the proposed computer model, with the use of characteristics of the centrifugal slurry pump GrAT-225-67-3-2-2. The said model of a centrifugal pump is efficient and can be used during the study of dynamic processes in pipeline transportation systems, as well as in the synthesis of control algorithms for electric drives of pumping units.
\end{abstract}

\section{Introduction}

One of the methods for controlling the flow of slurry is to control the electric motor speed. Frequency-controlled electric motor drive can significantly reduce electric power consumption, due to the possibility of using large pumping units at low flows [1, 2]. This method has long, been used, but due to the fact, that slurry pumped in the pipeline has an unstable ratio of solid and liquid phases $[3,4]$, it is necessary to take into account the change in slurry concentration in the control algorithm. While maintaining the concentration constant, the operating point of characteristics of the pump and network will be in the same position with minimal deviations [5]. This mode of operation corresponds to constant power consumption, which is economically viable $[6,7]$.

To develop new control algorithms for controlling slurry pumps, it is necessary to have a reliable mathematical model of their operation, convenient for computer simulation implementation.

The model should provide the possibility to simulate transient processes in a system [8] that includes: a slurry pipeline, a centrifugal pump, equipped with a frequencycontrolled asynchronous electric motor drive and a control device for controlling their joint operation. It is necessary to be able to assess properly the energy processes in the elements of the system $[9,10]$, since one of the main tasks of improving the hydrotransport of mining enterprises is to increase the energy efficiency of their operation [11].

A package of the applied mathematical programs scilab with the extension for graphical simulation of xcos dynamic systems was chosen as the simulation environment. The choice is mainly conditioned by the fact that the package is a freeware one and, therefore, available for free to most researchers. Moreover, this software is sufficient to solve the research tasks. Nonetheless, if necessary, the developed models can be implemented with minimal changes in other software systems, in particular, in the MATLAB Simulink environment.

\section{Methods}

As is shown in [12], the natural Q-H characteristic of a pump can be approximated with adequate accuracy by a second-order polynomial using the least-squares method. Therefore, the equation of the pump characteristic can be expressed as follows

$$
H=H_{\mathrm{H} 0}-r_{\mathrm{H} 1} Q-r_{\mathrm{H} 2} Q^{2} \text {, }
$$

where $H$ is the head pressure, $\mathrm{m} ; \mathrm{Q}$ is the volumetric flow rate, $\mathrm{m}^{3} / \mathrm{s} ; H_{\mathrm{H} 0}$ is the apparent head pressure at a flow rate equal to zero, $\mathrm{m} ; r_{\mathrm{H} 1}$ and $r_{\mathrm{H} 2}$ are the linear and quadratic components of the apparent hydraulic resistance of the pump.

The same source states that, when controlling the pump rotor speed, expressions that allow determining the parameters of the artificial Q-H characteristic, are valid for the parameters (1)

$$
\begin{aligned}
& H_{\mathrm{H} 0}^{c}=\left(H_{\mathrm{H} 0} / \omega_{\mathrm{H}}^{2}\right) \omega^{2} \\
& r_{\mathrm{H} 1}^{\prime}=\left(r_{\mathrm{H} 1} / \omega_{\mathrm{H}}\right) \omega \\
& r_{\mathrm{H} 2}^{\prime}=r_{\mathrm{H} 2},
\end{aligned}
$$

\footnotetext{
* Corresponding author: voytuk_irina@mail.ru
} 


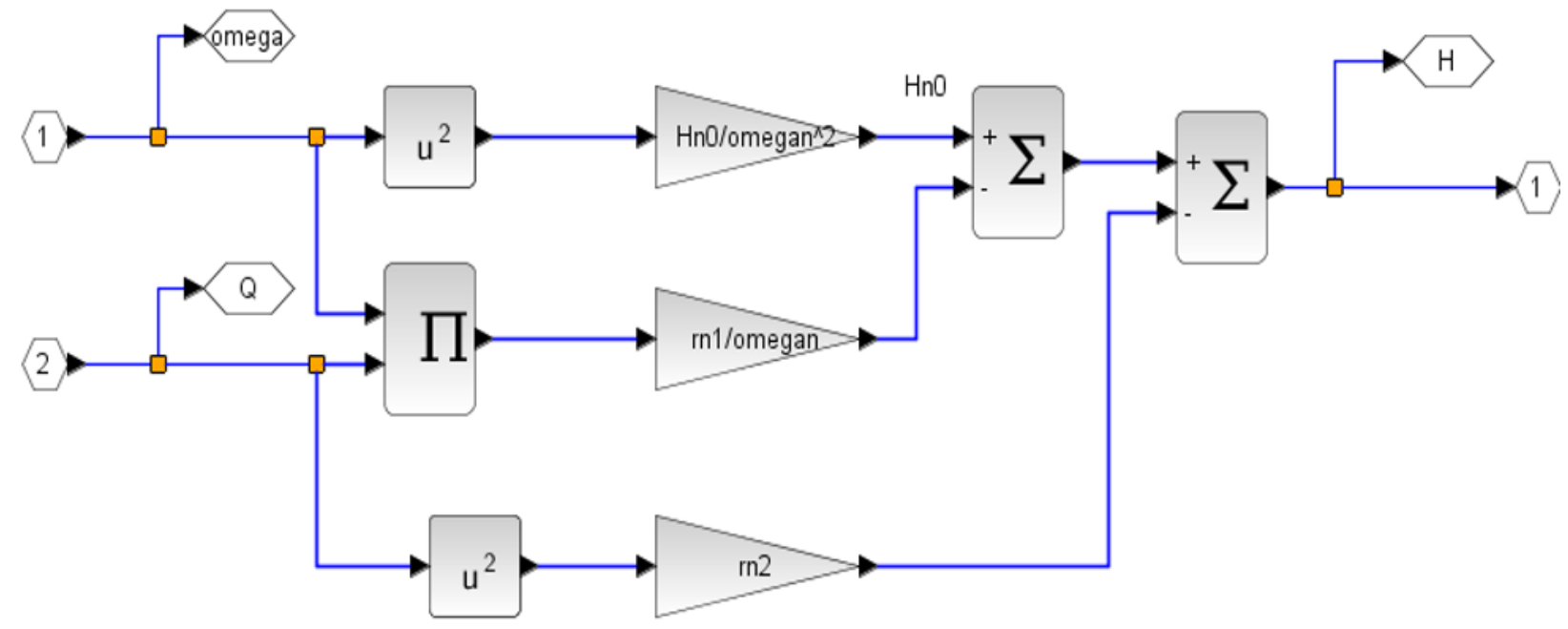

Fig. 1. Caption of the Figure 1. Below the figure.

where $\omega$ is the pump rotor speed, $\mathrm{rad} / \mathrm{s} ; \omega_{\mathrm{H}}$ is the pump rotor rated speed, $\mathrm{rad} / \mathrm{s}$.

Expressions (1) - (4) correspond to the xcos scilab diagram, shown in fig. 1.

In this model, the pump is considered as a source of head pressure, the value of which depends on the volumetric flow rate of the pumped fluid. The rotor speed signal is used as the model input signal.

In order to ensure the interaction between the pump model and the electric drive motor model, as well as to assess energy processes, it is necessary to supplement the model by calculating the mechanical power consumed by the drive motor, as well as by calculating the moment of resistance to the drive motor rotation.

The mechanical power (W) of a centrifugal pump can be calculated using the known formula [13]

$$
P_{\mathrm{H} 0}=\rho g H Q / \eta_{\mathrm{H}},
$$

where $\rho$ is the pumped fluid density, $\mathrm{kg} / \mathrm{m}^{3} ; g$ is the gravity acceleration, $\mathrm{m} / \mathrm{s}^{2} ; \eta_{\mathrm{H}}$ is the pump efficiency factor.

The anti-torque moment can be calculated by the formula [14]

$$
M=P_{\mathrm{H}} / \omega .
$$

Expressions (5) and (6) correspond to the xcos scilab diagram, shown in fig. 2. At the input of the division blocks, small constants are added to the input signals, corresponding to the dividend, which are necessary to avoid division by zero at the beginning of the simulation.

The efficiency factor, in the general case, depends on the rotor speed and on the pumped fluid flow rate. These dependencies can be given in the catalogued data, however, in a first approximation the efficiency factor, as shown in [15], can be calculated by the formula

$$
\eta=\eta_{H}\left(1-k\left[1-\left(\omega / \omega_{\mathrm{H}}\right)^{2}\right]\right)\left(1-\left[1-\left(Q / Q_{\mathrm{H}}\right)^{\mathrm{a}}\right],\right.
$$

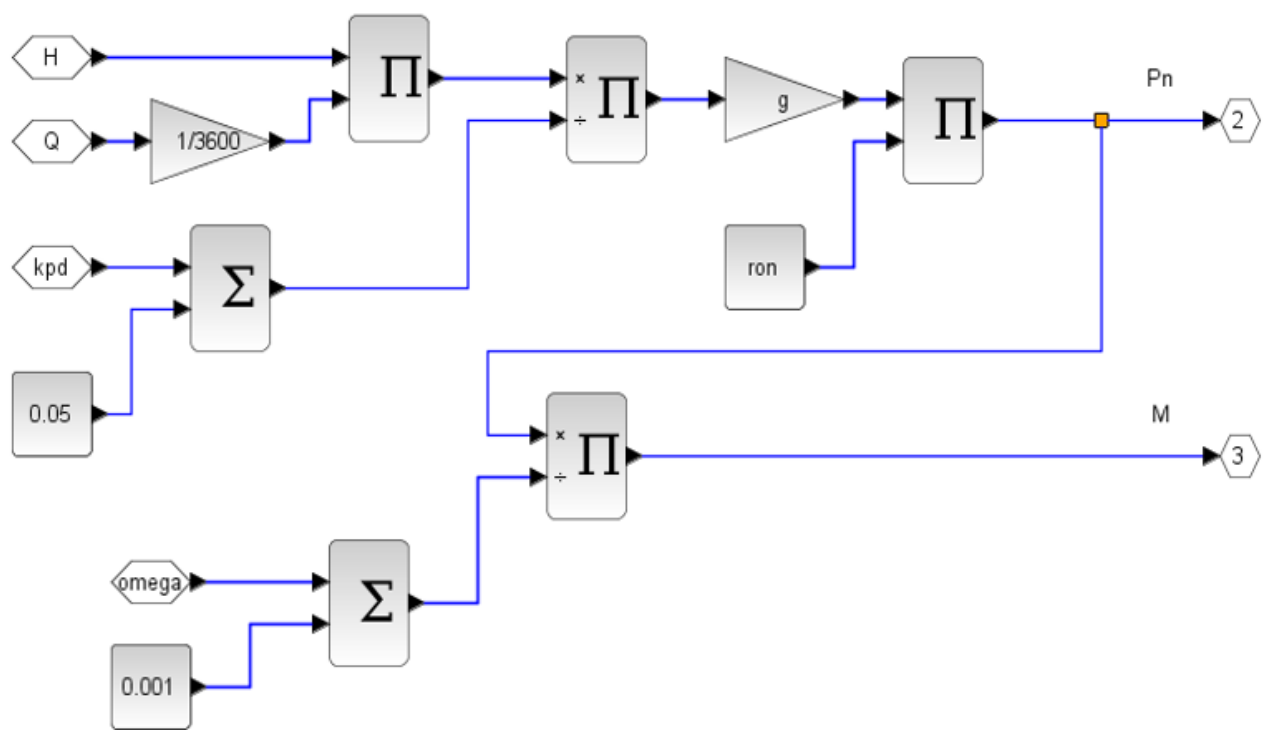

Fig. 2. Model of computing the pump power and the anti-torque moment. 


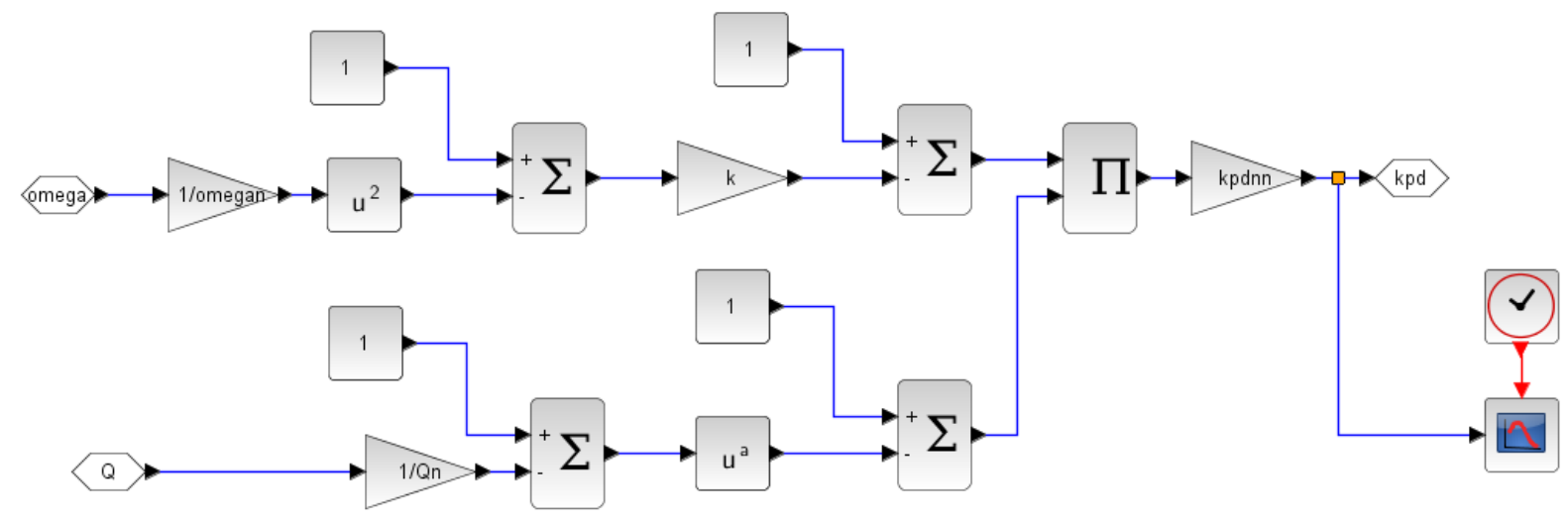

Fig. 3. Model of computing the pump efficiency factor.

where $\eta_{H}$ is the rated value of the pump efficiency factor; $a, k$ are constant coefficients depending on the characteristics of a separate pump, which can either be obtained by the method of approximation of operating characteristics, or found in the reference literature.

Expression (7) corresponds to the xcos scilab diagram, shown in fig. 3.

The diagrams, shown in fig. 1-3, together create a model of a centrifugal pump, the input signals for which are the rotor speed and flow rate, and the output signals are head pressure, mechanical power and the moment of resistance to the rotor rotation.

The pipeline model in the simplest case can be derived from the known expression

$$
H=H_{\mathrm{c} 0}+r_{\mathrm{c}} Q^{2}
$$

where $H_{\mathrm{c} 0}$ is the static head in the pipeline, $\mathrm{m} ; r_{\mathrm{c}}$ is the hydraulic resistance of the pipeline.

However, when using the model, obtained in accordance with expression (8) and the previously mentioned model, the so-called "algebraic loop" occurs, since the set of equations (1) and (8) does not explicitly contain time constants. It is proposed to introduce into expression (8) a small time constant $T_{\mathrm{c}}$, the value of which is chosen to be much less than the electric motor drive mechanical constant, which includes the moment of inertia of the electric motor and the moment of inertia of the pump reduced to its shaft. In this case, expression (8) takes the form

$$
H=H_{\mathrm{c} 0}+r_{\mathrm{c}} Q^{2}+T_{\mathrm{c}} d Q / d t .
$$

Expression (9) corresponds to the xcos scilab diagram, shown in fig. 4.

In addition to the pressure input, there is an additional input, providing break in the feedback. This input is necessary to simulate the pump starting, which is performed against closed valve gate, in this case, the transported fluid flow rate is equal to zero, and the feedback signal, as entered the integrator input, must be equal to zero. As the valve gate opens, this control signal will gradually increase to unity; at the end of the valve gate opening, the model will work strictly in accordance with expression (9).

To verify the model adequacy, the simulation of Q-H

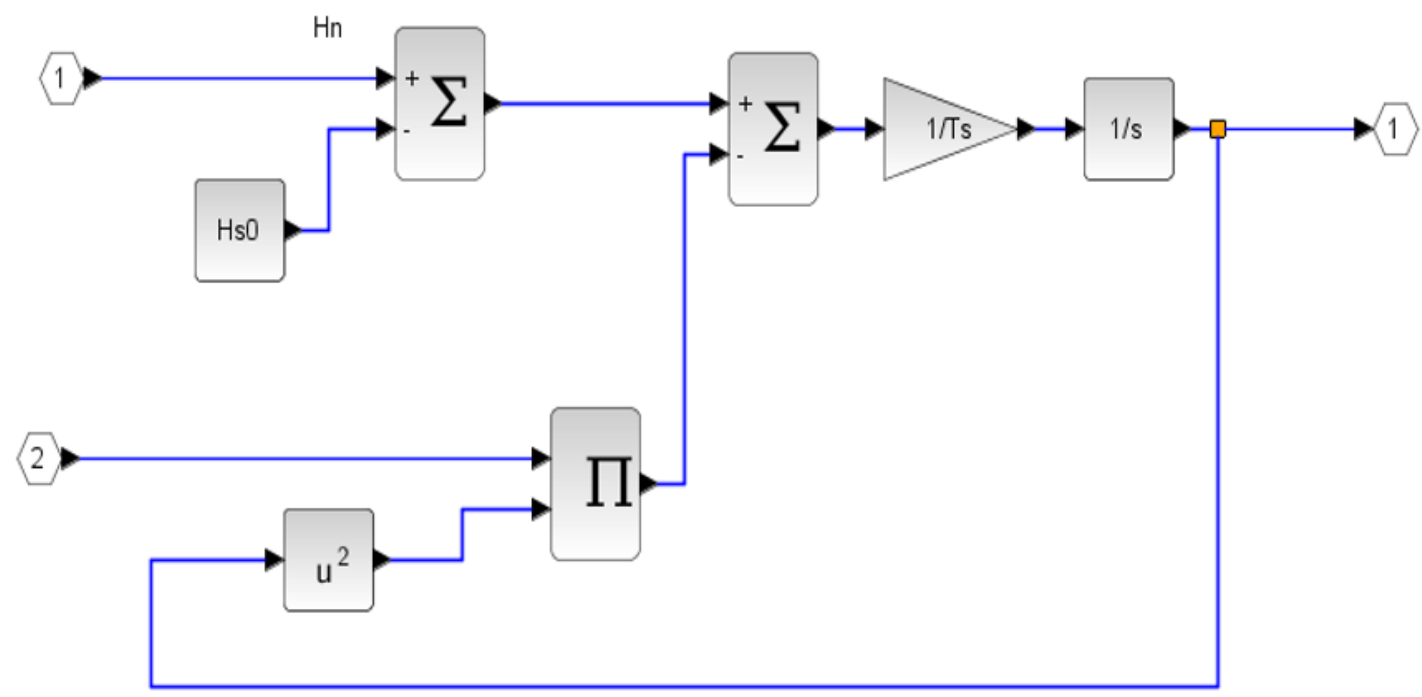

Fig. 4. The model of the pipeline. 


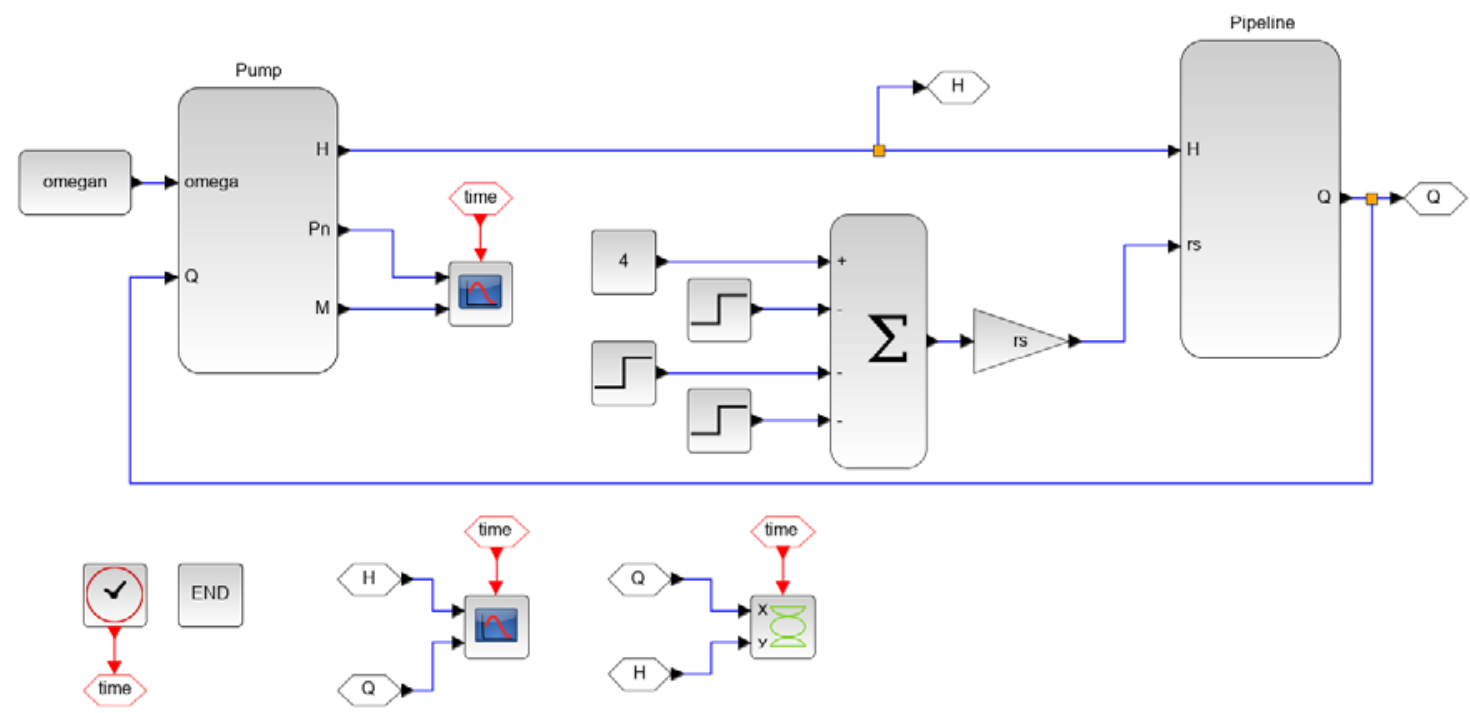

Fig. 5. The model for constructing Q-H characteristics of the slurry pump GrAT -225-67-3-2-2.

characteristics of the centrifugal slurry pump GrAT-22567-3-2-2 was performed, as shown in fig. 5. The pumped fluid density is taken equal to $2200 \mathrm{~kg} / \mathrm{m}^{3}$.

During the simulation, the pipeline hydraulic resistance was changed at various values of the speed of rotation. As an example, the characteristics were constructed for the rated speed of rotation (fig. 6a) and for the speed equal to 0.9 of the rated one (fig. $6 \mathrm{~b}$ ). The diagrams correspond to those given in the slurry pump technical passport, which witnesses in favor of the model adequacy.

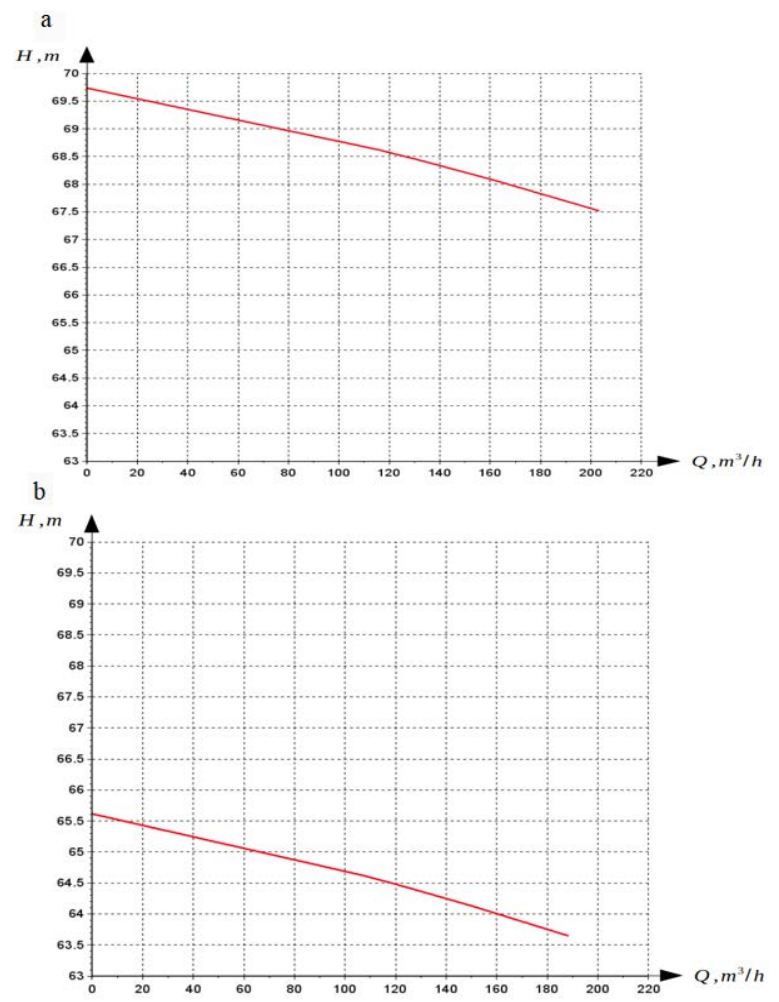

Fig. 6. Q-H characteristics of the GrAT -225-67-3-2-2 slurry pump at rated (a) and reduced (b) speed of rotation.

\section{Results and Discussion}

To study the model operation under dynamic mode, a model of the closed loop system for controlling head pressure (pressure) at the output of a slurry pump was developed (fig. 7). The model includes a model of an electric drive motor 4A315M4 with a frequency converter. The motor model is made in a fixed system of coordinates according to the known equations given in [16]. The motor model internal structure description is given in [17]. The model of the frequency converter is made similarly to the one given in [18]. It generates sinewave voltage signals along the axes $\alpha$ and $\beta$, the frequency and amplitude of which correspond to the specified ones, formed according to the law of scalar control [19, 20].

$$
U / f^{2} \rightarrow \text { const. }
$$

A number of additional blocks are used to form a smoothly increasing the velocity signal under start-up conditions, a signal for opening the valve gate after the pump creates the rated head pressure, and a signal for starting the controller upon completion of transient processes. Stepwise variation in the pipeline hydraulic resistance is used as a disturbance.

As a result of the simulation, transient curves were obtained, shown in fig. 8-13.

The following operating modes were simulated. In the time interval from 0 to $2 \mathrm{~s}$, the smooth starting of the slurry pump against the valve gate occurs. In this case, the pump accelerates to a speed close to ideal no-load speed. At the time point of 2 seconds, the valve gate starts to open and the load on the drive increases to the rated value within 2 seconds, wherein the speed decreases to the rated value, the head pressure and flow rate also correspond to the rated mode of operation. Then, the head pressure (pressure) controller is turned on and the head pressure decreases to the predetermined 


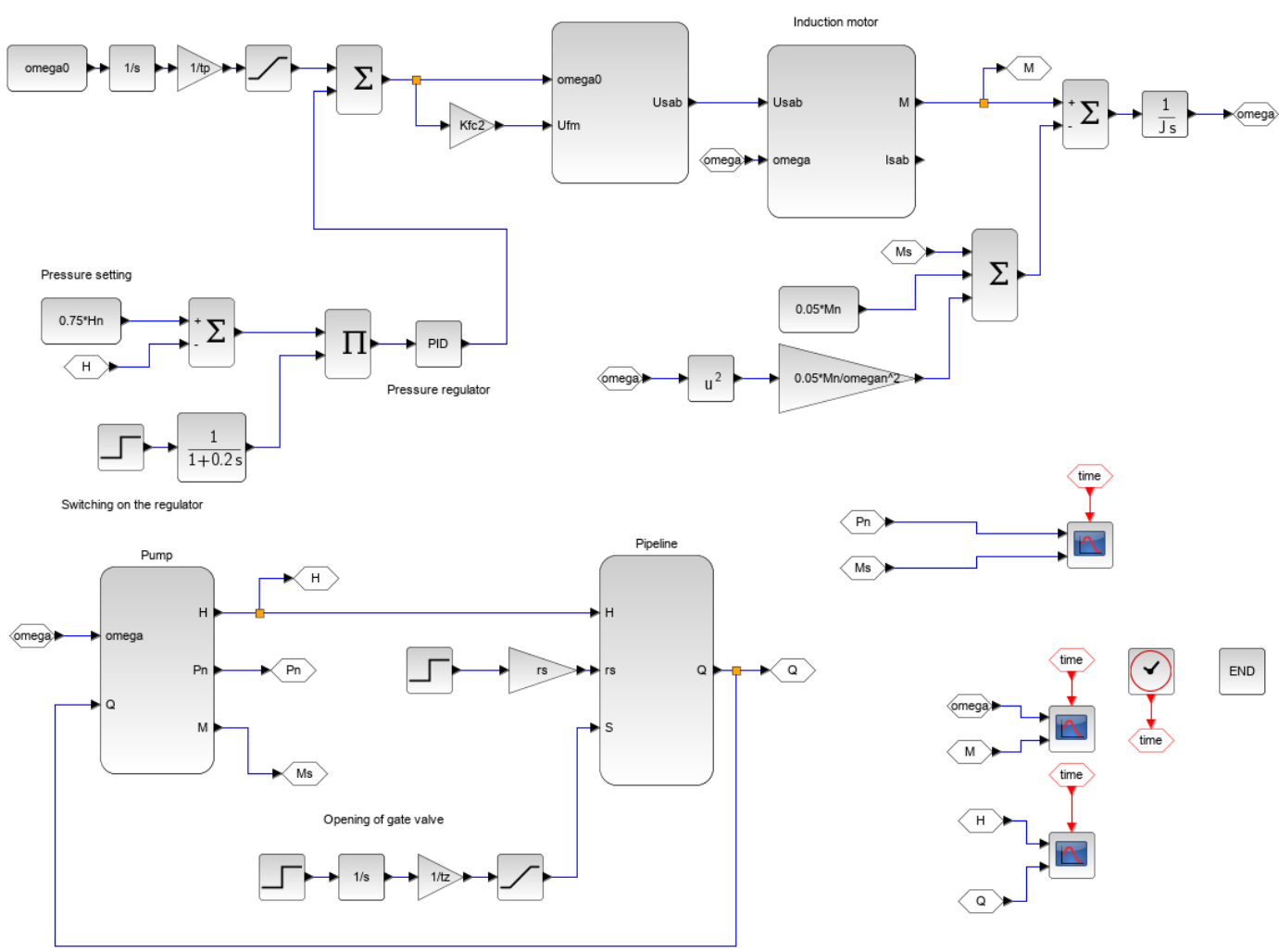

Fig. 7. Model of a closed loop system of a controlled motor drive.

value $-75 \%$ of the rated value, while the flow rate also decreases. At a time point of 8 seconds, a disturbance is formed in the form of a stepwise increase in the pipeline hydraulic resistance. From the diagrams it can be seen that the controller interpolates the disturbance, the speed, flow rate and load on the drive increase, and the head pressure returns to the predetermined value.

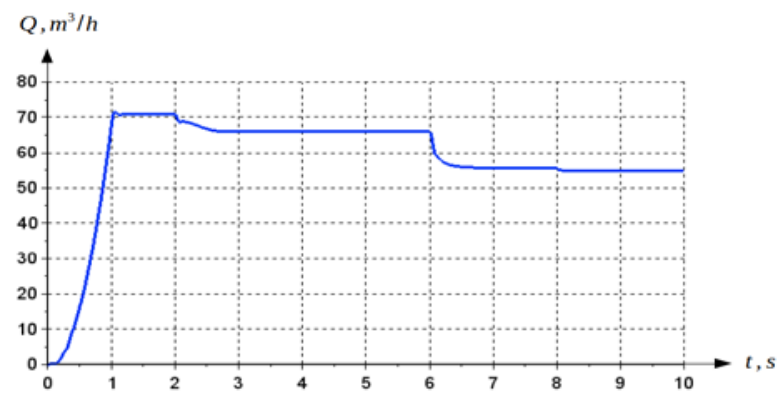

Fig. 8. Diagram of variation in flow rate.

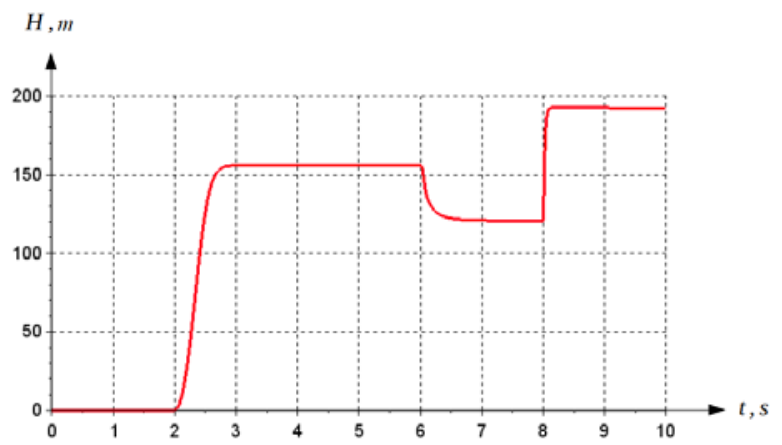

Fig. 9. Diagram of variation in head pressure.

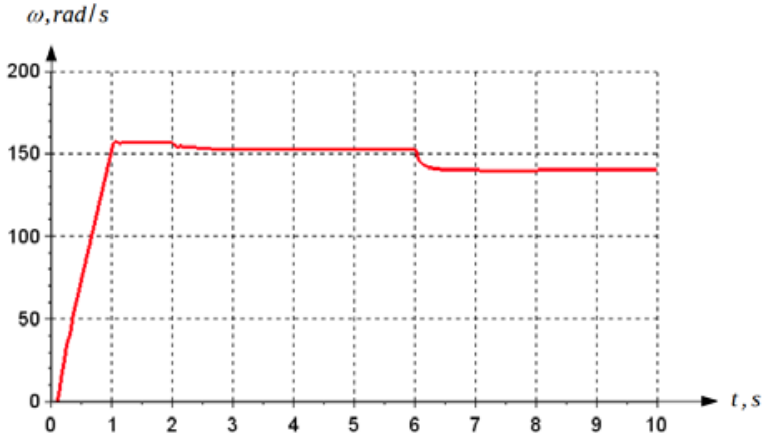

Fig. 10. Diagram of variation in rotor speed.

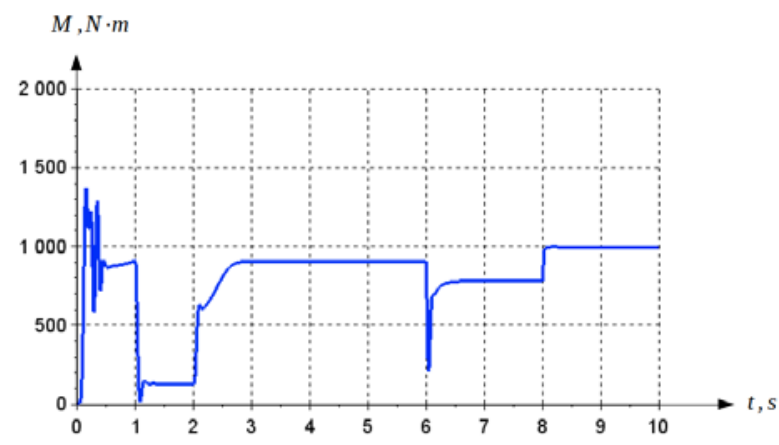

Fig. 11. Diagram of variation in torque on the drive motor shaft. 


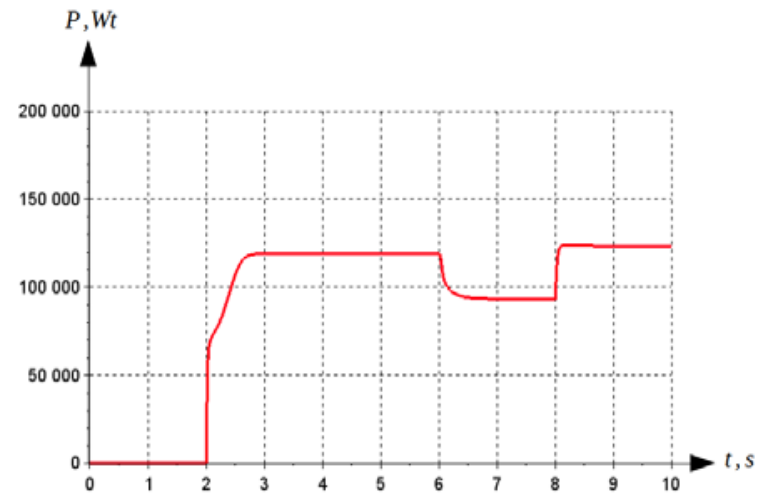

Fig. 12. Diagram of variation in mechanical power.

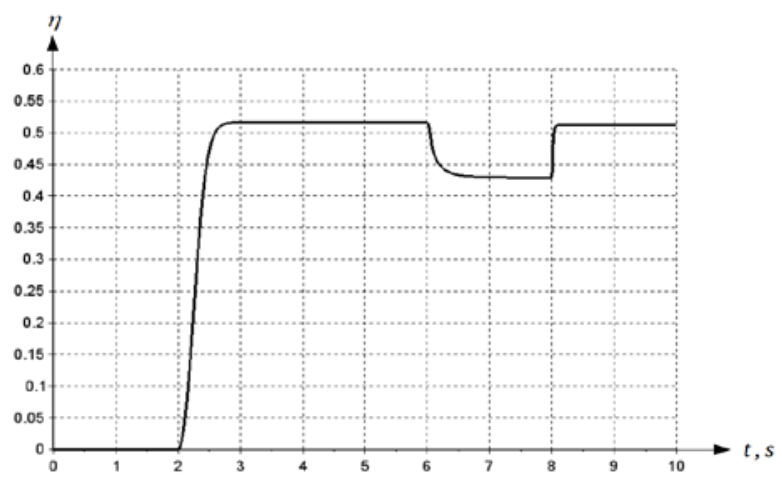

Fig. 13. Diagram of variation in the pump efficiency factor.

\section{Conclusions}

Summarizing the above, it can be concluded that the nature of the transient processes in the electric drive model of the slurry pump corresponds to physical processes. The main values, characterizing the processes in the slurry pipeline and pumping unit also vary under normal operating ranges. From the above it follows that the said model of a centrifugal pump is efficient and can be used during the study of dynamic processes in pipeline transportation systems, as well as in the synthesis of control algorithms for electric drives of pumping units.

The developed model accounts for the processes in both hydromechanical and electrical elements of the hydrotransport system, considers energy processes and allows one to implement various control algorithms, including those that account for variation in the physical and mechanical properties of the pumped slurry, its density and concentration. The proposed model can also be used during the study of dynamic transient processes in systems of controlled electric drive of centrifugal pumps of different purpose, as well as in the synthesis of their control systems.

\section{References}

1.R. Lawrence and C. Heron, in 2016 IEEE Pulp, Pap. For. Ind. Conf. (IEEE, 2016), 174-181 (2016)
2.S. Rachev, L. Dimitrov, K. Karakoulidis, I. D. Ivanov, and C.-V. Anghel Drugarin, in 2018 Int. Conf. Appl. Theor. Electr. (IEEE, 2018), 1-6 (2018)

3. E. Ikonen and P. Heikkinen, Neural Comput. Appl. 9, 165 (2000)

4.N. P. Ovchinnikov, Journal of mining institute. 235, 65 (2019)

5.P. M. Widodo and D. Rinaldy, J. Eng. Sci. Technol. 14, 1055 (2019)

6. A. Chusov, G. Podporkin, M. Pinchuk, D. Ivanov, I. Murashov, and V. Frolov, in 2016 33rd Int. Conf. Light. Prot. (IEEE, 2016), 1-9 (2016)

7. I. Murashov, V. Frolov, and D. Ivanov, in 2016 IEEE NW Russ. Young Res. Electr. Electron. Eng. Conf. (IEEE, 2016), 625-628

8. O. B. Shonin, V. S. Pronko, Journal of mining institute. 218, 270 (2016)

9. N. V. Obraztsov, D. I. Subbotin, V. E. Popov, V. Y. Frolov, and A. V. Surov, J. Phys. Conf. Ser. 1038, 012137 (2018)

10. I. S. Churkin, D. Ivanov, V. Frolov, and D. Uhrlandt, in 19th Symp. Phys. Switch. Arc 2011, FSO 2011 (2011)

11. V. I. Aleksandrov and Jerzy Sobota, Journal of mining institute. 213, 9 (2015)

12. R. Tao, R. Xiao, and W. Liu, Proc. Inst. Mech. Eng. Part A J. Power Energy (2018)

13. H. Sun, S. Yuan, Y. Luo, and Y. Guo, Paiguan Jixie Gongcheng Xuebao/Journal Drain. Irrig. Mach. Eng. (2016)

14. Y. D. Khechuev, B. E. Kalashnikov, and V. I. Ol\&apos;shevskii, Russ. Electr. Eng. (2006)

15. M. Zagirnyak, Przegląd Elektrotechniczny 1, 106 (2019)

16. K. A. Tahboub, M. I. Albakri, and A. M. Arafeh, in Vol. 4 ASME/IEEE Int. Conf. Mechatron. Embed. Syst. Appl. 19th Reliab. Stress Anal. Fail. Prev. Conf. (ASME, 2007), 209-217 (2007)

17. L. H. de Paula, F. C. Storti, and E. Fortaleza, IFAC-PapersOnLine 48, 33 (2015)

18. A. Makarov and M. Kukhtik, in 2018 Int. Ural Conf. Green Energy (IEEE, 2018), 265-269 (2018)

19. Z. B. Jiang, T. Zhong, and Y. H. Rao, in 2011 Int. Conf. Inf. Technol. Comput. Eng. Manag. Sci. (IEEE, 2011), 131-135 (2011)

20. Artyukhov, I. I. Bochkareva, and S. V. Molot, in 2014 Int. Conf. Actual Probl. Electron Devices Eng. (IEEE, 2014), 11-17 (2014) 